\title{
Involvement of CD147 isoform-4 in the proliferation of SiHa cells: A possible molecular mechanism of cervical cancer
}

\author{
YI WU ${ }^{1,3^{*}}, \mathrm{XI} \mathrm{ZHOU}^{1,4^{*}}$ and PENG-SHENG ZHENG ${ }^{1-3}$ \\ ${ }^{1}$ Department of Reproductive Medicine, First Affiliated Hospital of the Medical School of Xi'an Jiaotong University; \\ ${ }^{2}$ Division of Cancer Stem Cell Research, Key Laboratory of Environment and Genes Related to Diseases of the Ministry \\ of Education and ${ }^{3}$ Department of Biochemistry and Molecular Biology, Medical School of Xi'an Jiaotong University, \\ Xi'an, Shaanxi; ${ }^{4}$ Department of Obstetrics and Gynecology, Affiliated Renmin Hospital \\ of Hubei Medical University, Shiyan, Hubei, P.R. China
}

Received April 12, 2011; Accepted May 16, 2011

DOI: $10.3892 /$ or.2011.1345

\begin{abstract}
CD147, a multifunction glycoprotein of the immunoglobulin family, is associated with many types of tumors, and the overexpression of CD147 can enhance malignancy. However, the role of CD147 in cervical cancer remains unclear. In the present study, cervical cancer cells were found to express much more CD147 protein and isoform-4 than normal cervical cells $(\mathrm{P}<0.05)$, suggesting that $\mathrm{CD} 147$ and isoform-4 are involved in cervical cancers. Furthermore, we overexpressed the CD147 isoform-4 (CD147-4) in the cervical cancer cell line SiHa by transfection and found that CD147-4 promoted the proliferation of SiHa cells with a considerable proportion of cells in the $\mathrm{S}$ phase in which the expression of CIP2A, PLK and cyclin D1 was up-regulated while the expression of P27 was downregulated. This may be one of the molecular mechanisms involved in cervical cancer progression by CD147.
\end{abstract}

\section{Introduction}

CD147 is also called extracellular matrix metalloproteinase inducer (EMMPRIN), basigin or collagenase stimulatory factor $(1,2)$. It is a highly glycosylated cell surface transmembrane protein belonging to the immunoglobulin superfamily (3). CD147 is highly expressed in several types of human tumors, suggesting that CD147 plays a key role in tumor growth, invasion, metastasis and angiogenesis $(4,5)$. CD147-expressing cancer cells stimulate stromal cells to increase the production of vascular endothelial cell growth factor (VEGF) via the PI3K-Akt signaling pathway (6) and to secrete a high level of

Correspondence to: Professor Peng-Sheng Zheng, Department of Reproductive Medicine, First Affiliated Hospital of Medical School of Xi'an Jiaotong University, Xi'an, Shaanxi 710061, P.R. China E-mail: zpsheng@mail.xjtu.edu.cn

*Contributed equally

Key words: cervical cancer, CD147, isoform, proliferation, cell cycle several matrix metalloproteinases (MMPs) (7). This implicates the role of CD147 in several aspects of tumor progression, including local invasion and distant metastasis, due to the ability to degrade various extracellular matrix proteins. In addition, CD147 modulates cell proliferation serving as a receptor of cyclophilin A (8) while depletion of CD147 results in changes in AMP-activated protein kinase (AMPK) and mammalian target of rapamycin (mTOR) pathways, leading to diminished proliferation $(9,10)$. Although CD147 has been studied in many tumors, the role of CD147 in cervical cancer remains unclear.

Cervical cancer is the second most common cause of cancer-related mortality in women worldwide (11). One of the necessary agents in the development of cervical carcinoma is human papillomavirus (HPV) infection (12). Other established risk factors include smoking, multiple sexual partners, hormonal contraceptives and infection with other sexually transmitted diseases (13). There are approximately 500,000 new cases of cervical cancer per year, and $80 \%$ occur in developing countries (11). Therefore, it is crucial to investigate the molecular mechanisms of cervical cancer.

In the present study, we examined the expression patterns of CD147 and its isoforms in cervical cancer tissues and cell lines. Furthermore, we determined the functional roles of exogenous CD147 isoform-4 (CD147-4) in cervical cancer cell line, SiHa. Moreover, the possible molecular mechanism involved in cervical cancer progression was also investigated.

\section{Materials and methods}

Cell lines, tissue microarray slides and clinical samples. Human cervical cancer cell lines (HeLa, SiHa and C-33 A) were obtained from the American Type Culture Collection (ATCC) and maintained in Dulbecco's modified Eagle's medium-high glucose (DMEM; Sigma, St. Louis, MO) with $10 \%$ fetal bovine serum (FBS; Invitrogen, Carlsbad, CA).

Tissue microarray slides used for immunohistochemical staining were purchased from Shaanxi Chaoyin Biological Co. Clinical samples were obtained from patients undergoing surgical resections at the First Affiliated Hospital of Xi'an 
Table I. Primer sequences for RT-PCR.

\begin{tabular}{llr}
\hline Symbol & \multicolumn{1}{c}{ Primer sequence } & Product length (bp) \\
\hline CD147-1 & Primer F: 5'-AGGAGGACACGGGCACTTA-3' & 197 \\
& Primer R: 5'-CTCTGTGGCGCTGTCATTCA-3' & 135 \\
CD147-2 & Primer F: 5'-CTGTTCGTGCTGCTGGGATT-3' \\
& Primer R: 5'-CTCTGTGGCGCTGTCATTCA-3' \\
CD147-4 & Primer F: 5'-ATGAAGCAGTCGGACGCGTCTC-3' & 211 \\
$\beta$-actin & Primer R: 5'-TGACAGGTGGCACGGACTCTGACTT-3' & 239 \\
& Primer F: 5'-ATCGTCACCAACTGGGACGA-3' & \\
& Primer R: 5'-TCCATCACGATGCCAGTGGT-3'
\end{tabular}

Jiaotong University after informed consent. Normal cervical tissues were obtained from uterine resections for hysteromyoma.

Hematoxylin and eosin-stained sections were used to determine the pathological and cytological diagnosis. Clinical stage and the histological classification were identified according to the criteria of the FIGO (International Federation of Gynecology and Obstetrics) classification system.

Immunohistochemistry and cytochemistry. Formalin-fixed paraffin-embedded cervical cancer tissues were subjected to the catalyzed signal amplification system kit (SABC; Boster, Wuhang, China) according to the manufacturer's instructions. The sections were incubated with a primary antibody (antiCD147 monoclonal antibody; R\&D, Minneapolis, MN) at a dilution of 1:200 after dewaxing, rehydration, antigen retrieval and endogenous peroxidase activity blocking. Subsequently, the sections were incubated with a biotinylated secondary antibody (conjugated affinity purified goat anti-mouse immunoglobulin G) for $15 \mathrm{~min}$, which was followed by an incubation with peroxidase-marked streptavidin for an additional $15 \mathrm{~min}$. The reaction was visualized with 3,3'-diaminobenzidine (DAB), and the nuclei were counterstained with hematoxylin. For immunocytochemistry, cells were seeded on polylysinecoated glass slides, cultured for 2 days, fixed with $4 \%$ paraformaldehyde and then processed as the tissue sections. Negative controls were prepared using the same procedure, but phosphate-buffered saline (PBS) was substituted as the primary antibody.

Three independent researchers examined the sections in 10 representative microscopic fields in order to evaluate the expression of CD147. CD147 expression was assessed by counting the brown staining on the cell membrane or cytoplasm. The results were evaluated by an immunostaining score (14), ranging from 0 to 4 . The score was obtained by multiplying the percentage of positive cells (from 0 to 100\%) at each intensity with the corresponding immunostaining

Tumorsphere formation and differentiation. Tumor cells obtained from clinical samples were resuspended and seeded in serum-free DMEM/F12 containing 1X B-27 supplement (Invitrogen), epidermal growth factor (EGF) and basic fibroblast growth factor (bFGF; PeproTech Inc., Rocky Hill, NJ) (15). Tumorspheres were cultured in DMEM/F12 containing $10 \%$ FBS for 10 days to induce differentiation.
Flow cytometry. The single-cell suspension was washed and resuspended in $100 \mu \mathrm{l}$ per $10^{6}$ cells. Subsequently, the cells were incubated in PBS containing $2 \%$ FBS and $0.1 \%$ sodium azide with fluorescein isothiocyanate (FITC)-conjugated antibody (mouse $\mathrm{IgG}_{1} \kappa$; BD, New York, NY) on ice for $30 \mathrm{~min}$ in the dark. Isotype-matched mouse immunoglobulins (BD) and PBS were used as the isotype and negative controls, respectively. Samples were analyzed by a FACSCalibur flow cytometer and CellQuest software (BD).

Cell cycle analysis. Cells $\left(1 \times 10^{6}\right)$ were harvested and washed with PBS, followed by fixation with $75 \%$ ethanol at $4^{\circ} \mathrm{C}$ overnight. After the cells were washed twice in PBS, the cells were resuspended in PBS with $50 \mu \mathrm{g} / \mathrm{ml}$ propidium iodide (PI; Sigma) and $10 \mu \mathrm{g} / \mathrm{ml}$ RNaseA (Sigma). They were then incubated at $4^{\circ} \mathrm{C}$ for $30 \mathrm{~min}$ in the dark. Cells were analyzed for DNA content by FACSCalibur, and the cell cycle phases were analyzed by CellQuest software.

Western blot analysis. Cells were extracted with ice-cold 1X lysis buffer with a cocktail of protease and phosphatase inhibitors. A total of $20 \mu \mathrm{g}$ protein from each supernatant with an equal volume of $5 \mathrm{X}$ sodium dodecyl sulfate (SDS) gel-loading buffer was boiled for $5 \mathrm{~min}$ and loaded on 10\% SDS-polyacrylamide gel for electrophoresis. After the proteins were transferred onto nitrocellulose membranes by electroblotting, the membranes were blocked in 5\% milk in Tris-buffer salineTween-20 (TBST) and then incubated with the primary antibody at $4^{\circ} \mathrm{C}$ overnight. Subsequently, the membranes were incubated with the horseradish peroxidase (HRP)-conjugated secondary antibody at the appropriate dilution. The membranes were visualized with Enhanced Chemiluminescence-SuperSignal West Pico Trial Kit (Pierce, Rockford, IL) and applied to X-ray film.

Polymerase chain reaction analysis (PCR). Total RNA from the cells and tissues was extracted with TRIzol reagent (Invitrogen), and first-strand cDNA was synthesized by M-MuLV reverse transcriptase and random hexamer primer (MBI, Hanover, MD) according to the manufacturer's instructions. PCR amplification was carried out with 30 cycles at an equal annealing temperature, and the amplicon was analyzed by electrophoresis on agarose gel. $\beta$-actin was used as the internal control. Table I lists all PCR primers. 


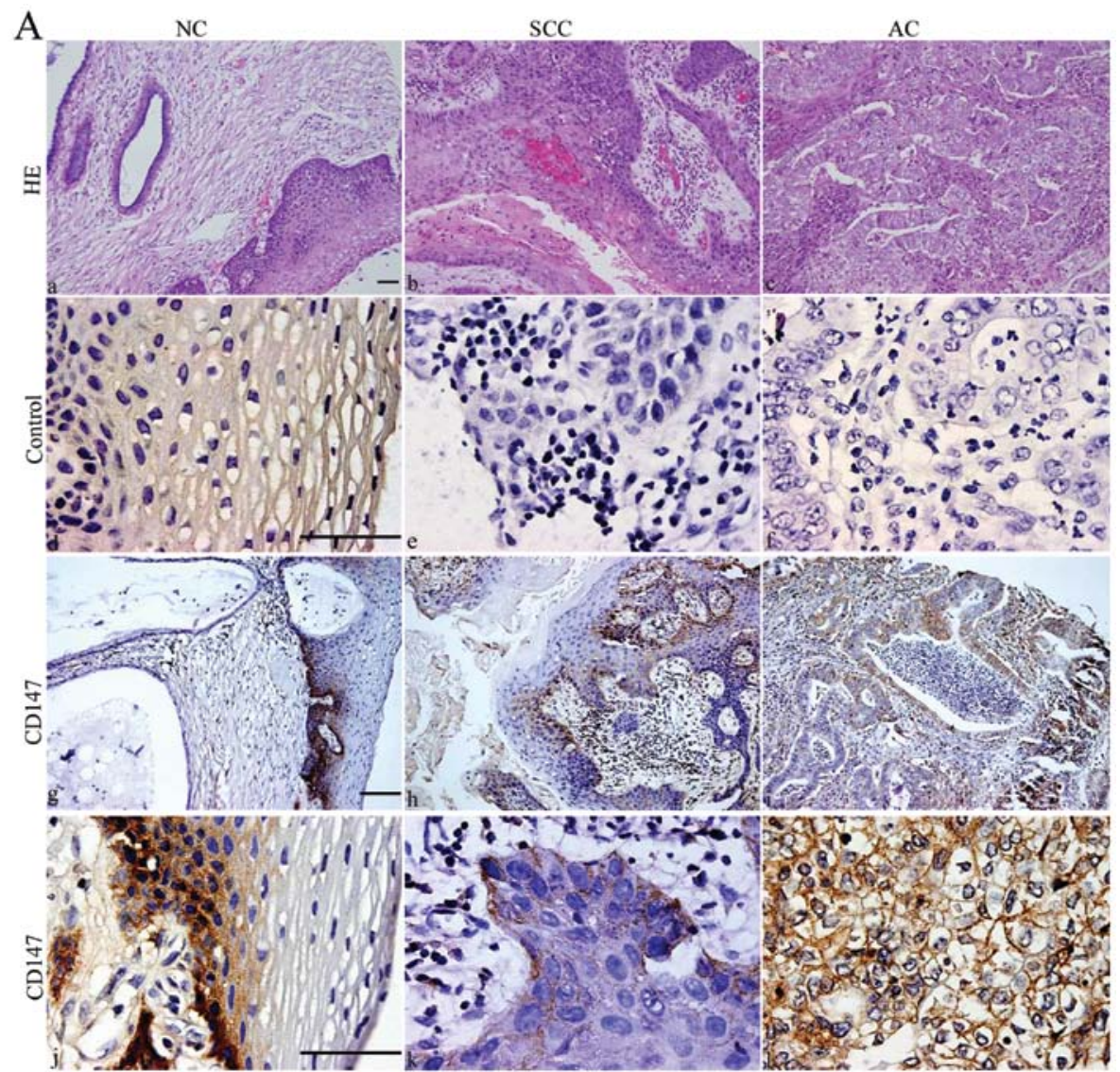

B

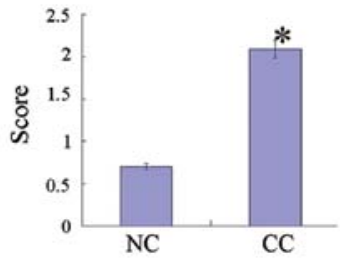

C

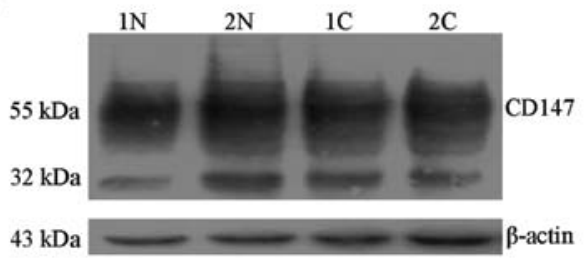

Figure 1. CD147 expression in normal cervical and cervical cancer tissues. (A) Hematoxylin and eosin staining (a-c, $x 200)$, negative control (d-f, $x 1,000)$ and immunohistochemical staining for CD147 protein ( $\mathrm{g}-\mathrm{i}, \mathrm{x} 400 ; \mathrm{j}-1, \mathrm{x} 1,000)$ in paraffin-embedded sections of normal cervical tissues (NC), squamous cell carcinoma (SCC) and adenocarcinoma (AC). Scale bar, $50 \mu \mathrm{m}$. (B) Bar graph shows the different levels of CD147 expression between cervical cancer tissues (CC) and normal cervical tissues ( $\mathrm{NC}),{ }^{*} \mathrm{P}<0.05$. (C) Western blot analysis detected the low glycosylated form (32 kDa) and high glycosylated form (55 kDa) of CD147 in normal cervical tissues and cervical cancer tissues. Only two representative cases of each tissue are showed.

CD147-4 construction and transfection. CD147-4 was amplified as a 618-bp fragment by RT-PCR with high fidelity polymerase Pfx (Invitrogen) from C-33 A cells. The PCR product was inserted into the HindIII and BamHI sites of a His coding vector to create the expression plasmid pcDNA3.1D-CD147-4 V5-His-TOPO. The pcDNA3.1D V5-His-TOPO vector without any insert was used as a negative control.

Transfection was performed with Lipofectamine 2000 transfection reagent (Invitrogen) according to the manufacturer's intsructions. After $24 \mathrm{~h}$ of transfection, cells were maintained in fresh DMEM medium containing $10 \% \mathrm{FBS}$ and $500 \mu \mathrm{g} / \mathrm{ml}$ of G418 (Calbiochem, Germany) for another 3 weeks. Stably transfected clones were isolated and identified by Western blotting.

Proliferation assay. The 3-(4,5-dimethylthiazol-2-yl)-2,5diphenyl tetrazolium bromide (MTT) assay was performed to evaluate cell proliferation ability. Cells were seeded onto a 96-well plate at a density of 1,000 cells per well. A total of $20 \mu \mathrm{l}$ sterile MTT solution ( $5 \mathrm{mg} / \mathrm{ml}$; Sigma) was added to each well at day 1, 3, 5, 7 and 9, and the cells were incubated at $37^{\circ} \mathrm{C}$ for $4 \mathrm{~h}$. Following the incubation, $150 \mu \mathrm{l}$ dimethyl sulfoxide (DMSO; Sigma) was added to each well for dye extraction. The dark-blue crystals of MTT-formazan were thoroughly dissolved by shaking the plates at room temperature for $10 \mathrm{~min}$. Spectrometric absorbance at $490 \mathrm{~nm}$ was measured by a microplate reader (Bio-Rad, Hercules, CA). Blank controls were added in medium.

Scratch wound migration assay. Cells were counted and maintained in a 35-mm plate until reaching $90 \%$ confluency. The confluent cell layer was wounded by a sterile $200-\mu 1$ pipette tip. The cells were then cultured in serum-free medium to ensure growth inhibition. The distances migrated by the cells were measured, and images were captured at the same position every $12 \mathrm{~h}$ for 4 days. 


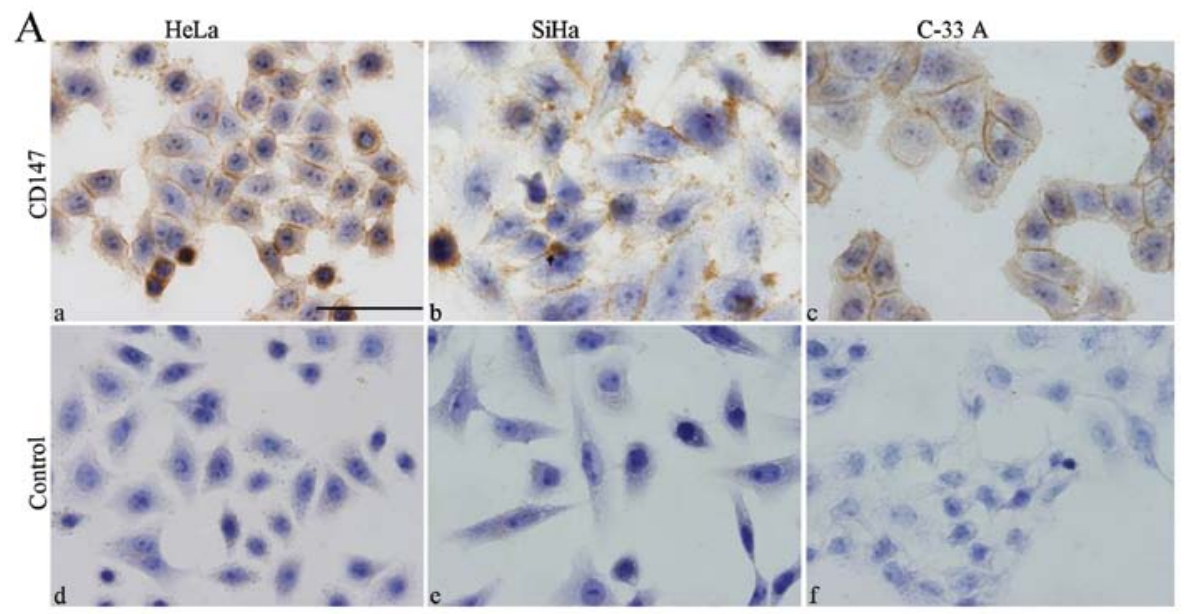

B
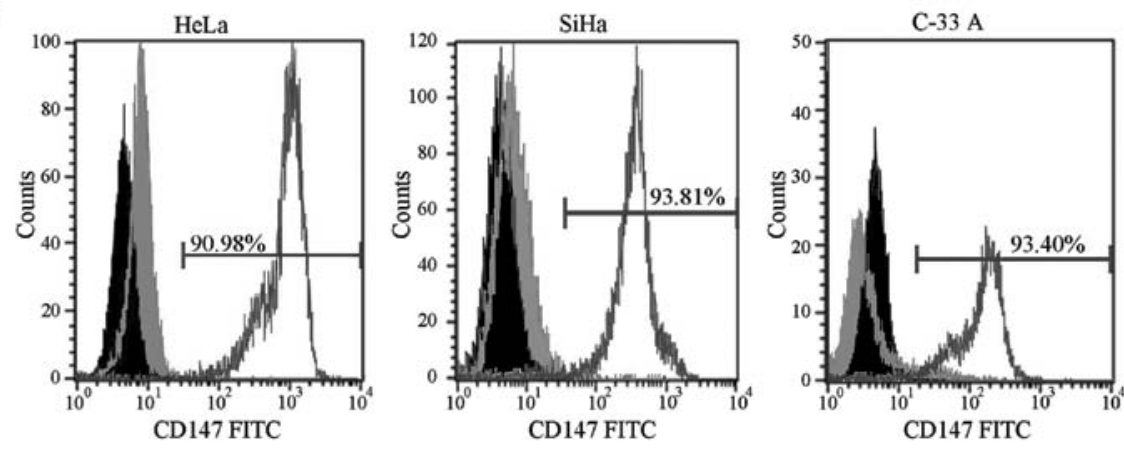

$\mathrm{C}$

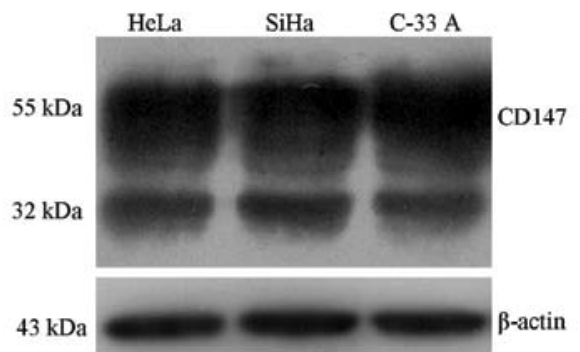

Figure 2. CD147 expression in cervical cancer cell lines. (A) Immunocytochemistry staining for CD147 protein (a-c, x1,000) and the negative control (d-f, x1,000) in the HeLa, SiHa and C-33 A cell lines. Scale bar, $50 \mu \mathrm{m}$. (B) The percentage of CD147-expressing HeLa, SiHa and C-33 A cells analyzed by flow cytometry. Grey-filled peaks show staining with FITC-conjugated isotype controls, black-filled peaks show negative controls of unstained cells, and empty peaks represent CD147-positive cells. (C) Western blot analysis detected two isoforms (32 and $55 \mathrm{kDa}$ ) of CD147 proteins in the HeLa, SiHa and C-33 A cells.

Statistical analysis. Data were presented as the mean \pm SD. Differences between mean values were analyzed by the Student's t-test or analysis of variance (ANOVA) followed by a post hoc multiple comparison. A P-value $<0.05$ was considered as statistically significant. All data were analyzed by SPSS 16.0 software (SPSS Inc., Chicago, IL).

\section{Results}

CD147 protein in different human cervical lesions. To analyze the CD147 expression in cervical cancers, we performed immunohistochemical staining using 27 normal cervices and 67 cervical cancer tissues. Fig. 1A shows that CD147 was expressed in most of the cervical cancer cells but only in basal cells of the normal cervical epithelia, and all positive staining was on the cell membrane. The percentage of positive cells was $73.51 \%$ in the cervical cancer tissues and $20.56 \%$ in the normal cervical tissues, whereas the immunostaining score was 2.09 and 0.70 (Table II), respectively. Therefore, the CD147 expression in the cervical cancer tissues was significantly higher than that in the normal cervical epithelial tissues $(\mathrm{P}<0.05)$ (Fig. 1B). Furthermore, we detected two bands $(\sim 32$ and $55 \mathrm{kDa}$ ) in both the cervical cancer and normal tissues by Western blotting, which were different glycosylated forms of CD147 (Fig. 1C).

CD147 protein in the cervical cancer cell lines. Fig. 2A shows that CD147 was peripherally expressed on the cell membrane with an intense immunoreactivity and a high frequency in three cervical cancer cell lines, HeLa, SiHa and C-33 A, by immunocytochemistry. However, no immunostaining was detected in the negative controls. Flow cytometric analysis showed that the percentage of positive cells was 90.98, 93.81 and $93.40 \%$ in HeLa, SiHa and C-33 A, respectively (Fig. 2B). 
Table II. Immunohistochemistry evaluation of CD147 protein.

\begin{tabular}{|c|c|c|c|c|}
\hline & No. & $\begin{array}{c}\text { Positive } \\
\text { percentage }^{\mathrm{a}}\end{array}$ & Level $^{\mathrm{b}}$ & Score $^{c}$ \\
\hline Normal cervix & 27 & 20.56 & $2.92 \pm 1.40$ & $0.70 \pm 0.60$ \\
\hline Cervical cancer & 64 & 73.51 & $2.66 \pm 1.13$ & $2.09 \pm 1.04$ \\
\hline P-value & & 0.000 & 0.379 & 0.017 \\
\hline
\end{tabular}

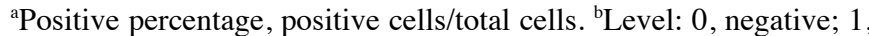
weak; 2 , moderate; 3 , intense; 4 , most intense staining. 'Score, positive percentage $\mathrm{x}$ level.

Furthermore, we detected two forms of CD147 in all three cell lines, which were the same as those detected in the cervical cancer tissues and normal uterine cervical tissues by Western blotting (Fig. 2C).

CD147 protein in cervical tumorspheres and differentiated cancer cells. To study whether CD147 is associated with tumorinitiating cells, we cultured the tumorspheres from primary cervical cancers in serum-free culture medium as previously described, and were then cultured in DMEM with 10\% FBS for cell differentiation (Fig. 3A). Tumorspheres are considered as an enrichment of tumor-initiating cells, which retain tumorigenic potential (16). However, our data did not indicate any significant difference in CD147 expression between the tumorspheres and the differentiated cells by flow cytometric analysis (Fig. 3B). CD147 was highly expressed in both (P>0.05) (Fig. 3C).

CD147 isoforms in cervical cancers. To explore the roles of different CD147 isoforms in cervical cancers (17), we designed three pairs of primers to detect the expression of three transcripts at the mRNA level (Fig. 4A). Fig. 4B shows the CD147 expression of individual isoforms in the cervical cancer and normal cervical tissues. CD147 isoform-2 (CD147-2) was extensively expressed in the cervical cancer tissues $(23 / 23$, $100 \%$ ) and normal cervical tissues (14/15, 93.3\%), while CD147 isoform-1 (CD147-1) showed a trend toward lower expression in the cervical cancer tissues $(14.3 \%)$ and normal cervical tissues $(33.3 \%)(\mathrm{P}>0.05)$. Moreover, Fig. 4C shows that CD147-4 was more frequently detected in cervical cancer tissues $(42.9 \%)$ than in the normal cervical tissues $(6.7 \%)(\mathrm{P}<0.05)$ (Table III). These results suggests that CD147-4 plays an important role in cervical cancers. CD147-4 was also expressed in the HeLa and C-33 A cell lines but absent in SiHa, while CD147-2 was expressed in all three cell lines and CD147-1 was expressed in C-33 A only (Fig. 5A).

Effects of exogenous CD147-4 on SiHa cells. To study the roles of CD147-4 in cervical cancers, we transfected the exogenous His-tagged CD147-4 in SiHa cells (Fig. 5B). MTT assay demonstrated that exogenous CD147-4 promoted the growth of SiHa cells $(\mathrm{P}<0.05)$ (Fig. 5C), and the proliferation rate of CD147-4-transfected SiHa cells was 2 -fold higher than that of the control cells at day 7. Furthermore, Fig. 5D shows that more cells in the S phase were found in the CD147-4-

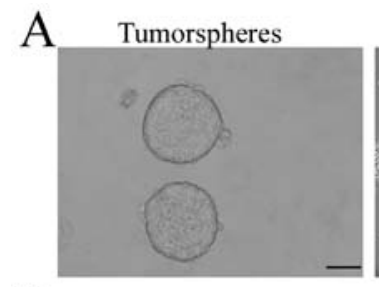

Differentiated cells
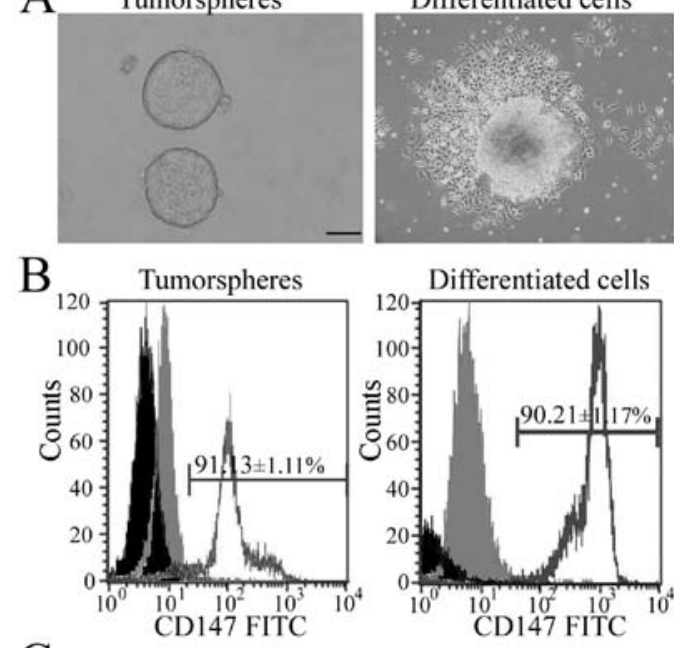

C

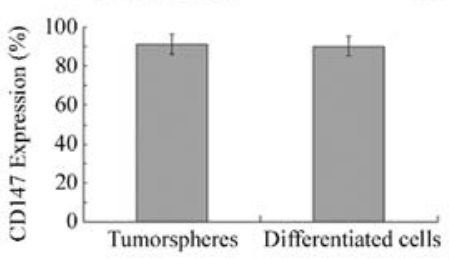

Differentiated cells

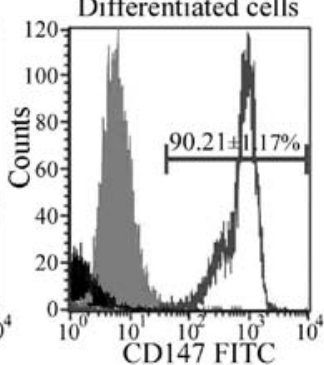

CD147 expression in tumorspheres and differentiated cancer cells. (A) Cervical cancer tumorspheres (left) cultured in serum-free condition (x200) and differentiated cancer cells (right) cultured in 10\% FBS DMEM (x200). (B) The percentage of CD147-expressing cells in cervical cancer tumorspheres and differentiated cancer cells analyzed by flow cytometry. (C) Bar graph shows no difference in CD147 expression between cervical cancer tumorspheres and differentiated cancer cells.

Table III. Expression of CD147 variants in the clinical samples.

\begin{tabular}{|c|c|c|c|c|c|c|c|}
\hline & \multirow[b]{2}{*}{ No. } & \multicolumn{2}{|c|}{ Variant 1} & \multicolumn{2}{|c|}{ Variant 2} & \multicolumn{2}{|c|}{ Variant 4} \\
\hline & & + & - & + & - & + & - \\
\hline $\begin{array}{l}\text { Normal cervical } \\
\text { tissue }\end{array}$ & 15 & 5 & 10 & 14 & 1 & 1 & 14 \\
\hline Cervical cancer & 21 & 3 & 18 & 21 & 0 & 9 & 12 \\
\hline P-value & & \multicolumn{2}{|c|}{0.171} & \multicolumn{2}{|c|}{0.417} & \multicolumn{2}{|c|}{0.019} \\
\hline
\end{tabular}

transfected SiHa cells $(41.0 \%)$ compared with that of the control group (24.1\%), whereas fewer cells in the $G_{0} / G_{1}$ phase were found in the CD147-4 transfected SiHa cells $(54.7 \%)$ compared with that of the control group $(66.6 \%)(\mathrm{P}<0.05)$. Moreover, Western blot analysis revealed that the CD147-4transfected SiHa cells expressed a higher level of the cancerous inhibitor of PP2A (CIP2A), polo-like kinase (PLK) and cyclin D1 compared with the control group, whereas a lower level of tumor suppressor P27 was detected in the CD147-4transfected SiHa cells (Fig. 5E).

Previous research has demonstrated that CD147 is involved in cancer invasion and metastasis by promoting the expression of MMPs and increasing cell migration. However, in the present study, exogenous CD147-4 neither promoted the expression of MMP2 or MMP9 (Fig. 5F) nor altered cell migration (Fig. 5G). 
A
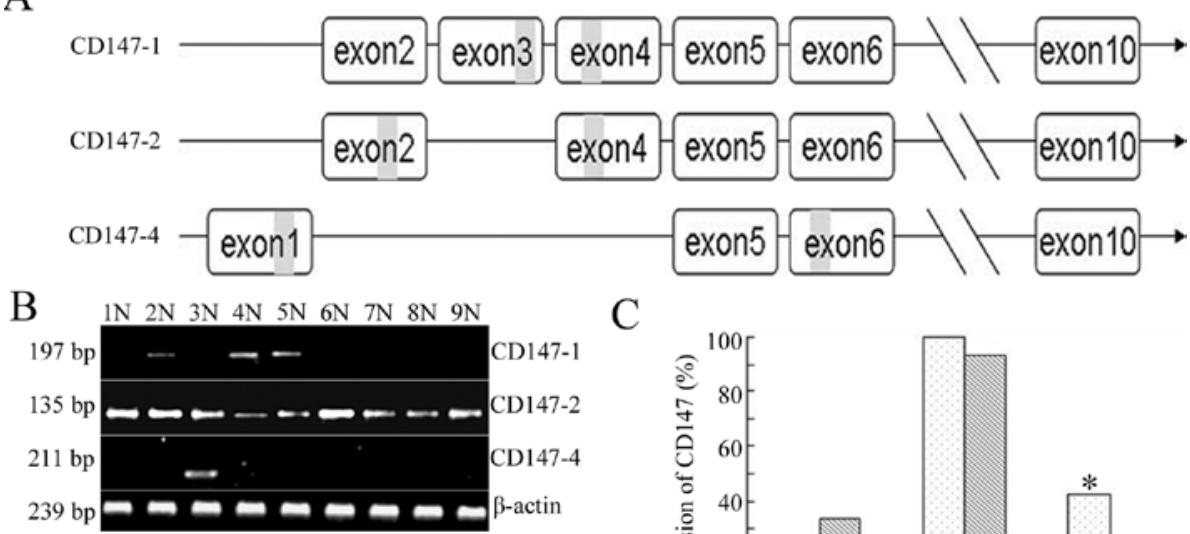

$\mathrm{C}$
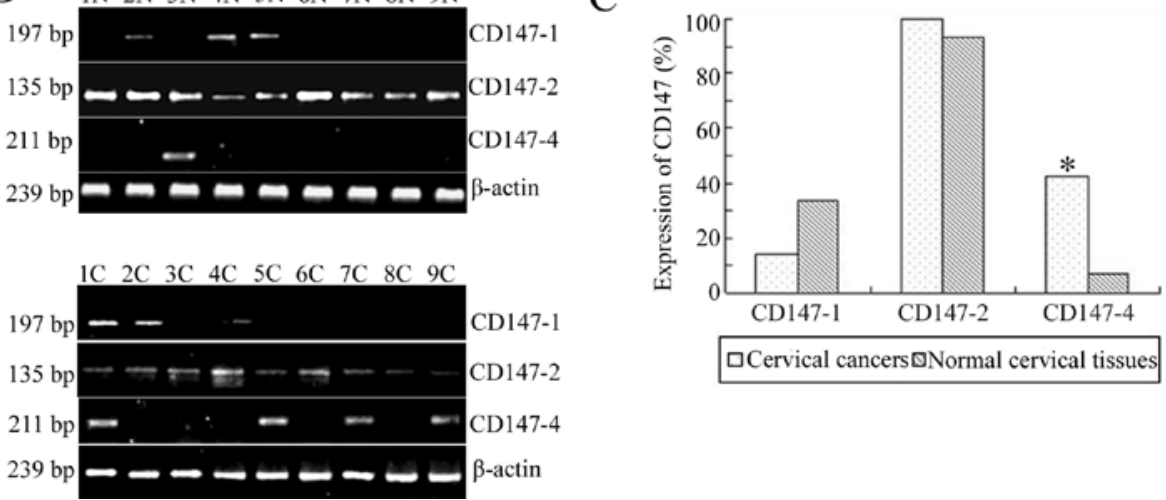

Figure 4. Expression of CD147 isoforms in normal cervices and cervical cancer tissues. (A) Structures of CD147 mRNA transcripts. Shaded regions represent the primer locations. (B) RT-PCR analysis of three CD147 isoforms in normal cervical tissues (upper panel) and cervical cancer tissues (lower panel). Nine representative cases of each tissue are shown. (C) The bar graph shows the different percentages of expression of three CD147 isoforms between cervical cancer tissues and normal cervical tissues; ${ }^{*} \mathrm{P}<0.05$.

A

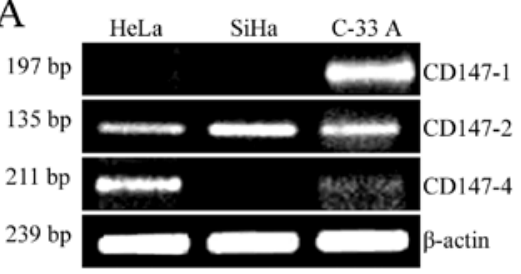

C

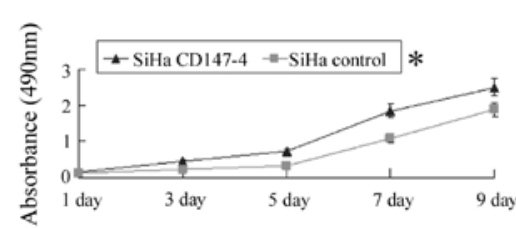

D

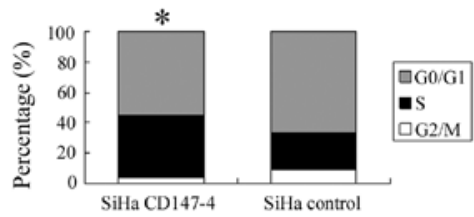

F

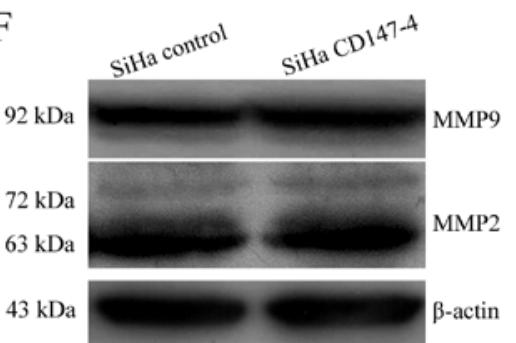

B

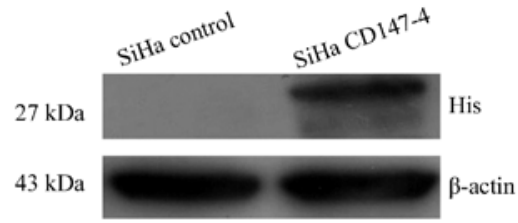

E

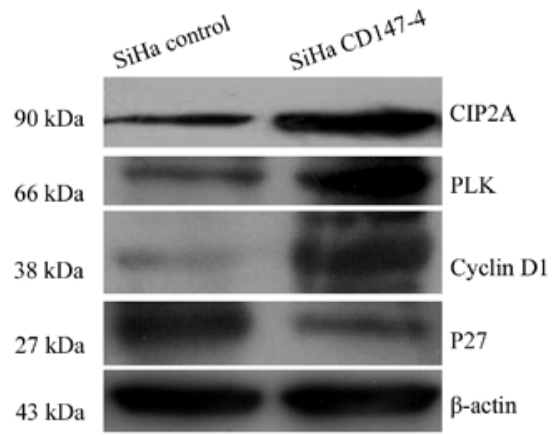

G

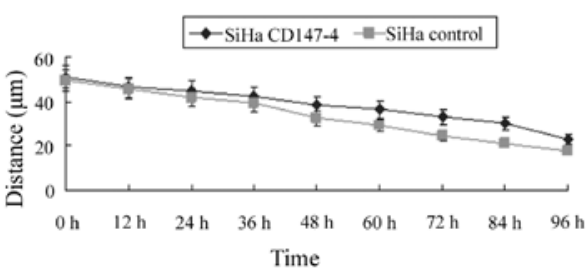

Figure 5. Effects of exogenous CD147-4 in SiHa cells. (A) RT-PCR analysis of the expression of CD147 isoforms in human cervical cancer cell lines: HeLa, $\mathrm{SiHa}$ and C-33 A. (B) Identification of SiHa CD147-4 clones by Western blot analysis with antibodies against HIS. (C) Effects of exogenous CD147-4 on the proliferation of SiHa cells by MTT assay. (D) Effects of exogenous CD147-4 on the cell cycle of SiHa cells by flow cytometry. The constituent ratio chart shows the proportion of cells in the $\mathrm{G}_{0} / \mathrm{G}_{1}, \mathrm{~S}$ and $\mathrm{G}_{2} / \mathrm{M}$ phases of the cell cycle; ${ }^{*} \mathrm{P}<0.05$. (E) Effects of exogenous CD147-4 on cell cycle proteins in SiHa cells by Western blot analysis. (F) Effects of CD147-4 on metastasis in SiHa by Western blot analysis. (G) The graph shows the distances migrated by the cells in the scratch wound migration assay. 


\section{Discussion}

CD147, a multifunction glycoprotein of the immunoglobulin family, is present in many types of normal cells and is involved in reproduction, embryo implantation, neural network formation and inflammation. Its overexpression in tumor cells promotes tumor invasion, metastasis and growth. The pathophysiological roles associated with these functions have been reported in several types of cancers, such as breast (18), glioma (19) and melanoma (20). In addition, a previous study found that CD147 is associated with cervical cancer and regarded as a prognostic factor in radiotherapy (21).

In the present study, we detected the expression of CD147 in cervical cancer tissues, normal cervical tissues and cervical cancer cell lines (Figs. 1 and 2). CD147 protein was abundant on the surface of cervical cancer cells, but it was only present on basal cells of normal cervices. Basal cells, considered as reserve cells, possess powerful proliferation ability and are presumed to be cervical stem cells (22). The location of CD147 on the basal layer of normal cervices suggests that CD147 may participate in the physiological function of the cervix and may be involved in the proliferation and initiation of cells. In addition, cervical cancer tissues had a significantly higher staining score than normal cervical epithelia which suggests that CD147 plays important roles in cervical cancers. Thus, we hypothesized that CD147 was associated with cervical cancer stem cells. Cancer stem cells are a rare cell population found in cancers, which contain the ability for self-renewal, differentiation and tumorigenesis (23). Previous research has demonstrated that tumorspheres are rich in cancer stem cells and give rise to tumors in vivo (16). In our study, however, both tumospheres and differentiated cells derived from fresh cervical cancer tissues highly expressed CD147 (Fig. 3), indicating that CD147 did not initiate the cervical cancers but promoted the progression by enhancing proliferation.

The low glycosylated form $(32 \mathrm{kDa})$ and the high glycosylated form $(55 \mathrm{kDa})$ of CD147 were identified by Western blot analysis. Glycosylation contributes to the different molecular weights of CD147 (24). N-glycosylation of CD147 is essential for invasion and metastasis of tumor, as only highly glycosylated-CD147 is responsible for MMP-stimulating activity. The deglycosylated CD147 not only fails to induce MMP activity but also antagonizes the MMP-inducing activity of glycosylation CD147 (25). Therefore, the cervical cancer and normal tissues expressed the functional form of CD147.

The CD147 gene is located on chromosome 19p13.3, and multiple mRNA transcripts encoding different protein isoforms have been reported. There are three representative transcripts, isoform 1, 2 and 4. The isoform-1 (NM_001728.2) represents the longest transcript and encodes the longest protein isoform, which contains two extracellular Ig-like domains, one highly conserved transmembrane domain (24 amino acids) and one cytoplasmic domain ( 40 amino acids). The differences between the isoforms mainly lie within the extracellular domains. Isoforms 2 (NM_198589.1) and 4 (NM_198591.1) contain only one Ig-like domain. Isoform-2 has the same $\mathrm{N}$ - and C-terminus as isoform- 1 , whereas isoform- 4 has a distinct $\mathrm{N}$-terminus. It has been reported that the expression of CD147-2 at the mRNA level is limited to the retina of mice, specifically to the photoreceptor cells (26), suggesting that CD147 is heterogeneously expressed and plays various roles in different tissues. To explore the functions of different CD147 isoforms in cervical cancers, we assessed the expression patterns of different isoforms in cervical cancer tissues and normal cervices (Fig. 4). RT-PCR analysis was applied since the CD147 antibody recognized the extracellular domains and failed to distinguish the different isoforms. The results showed that isoform- 4 may play an important role in cervical cancers, as it was expressed more often in cervical cancer tissues (42.9\%) than in the normal cervices $(6.7 \%)$.

In order to further identify the significance of CD147-4 in cervical cancer, we transfected CD147-4 in SiHa cells, which was negative for isoform- 4 . The results showed that CD147-4 promoted cell proliferation by up-regulating the expression of CIP2A, PLK and cyclin D1 and down-regulating the expression of P27 (Fig. 5). CIP2A is a human oncoprotein that inhibits the protein phosphatase 2A (PP2A) and dephosphorylation of c-Myc, thereby it stabilizes c-Myc and promotes malignant cell proliferation $(27,28)$. PLK serves as a marker of cell proliferation and is accumulated in the cell cycle $S$ and $\mathrm{G}_{2}$ phases. Therefore, PLK is closely related to polo and CDC5, which are involved in regulating the function of the mitotic spindle (29). P27 is a cyclin-dependent kinase inhibitor (CDKI), which prevents the activation of cyclin-CDK complexes and thus controls the cell cycle progression at $\mathrm{G}_{1}$. The degradation of P27 is required for the cellular transition from the quiescence to proliferative state. CD147-4 may enhance cell proliferation via augmenting these cell cycle regulators as well as inhibiting p27 tumor-suppressor protein.

It has been proven that CD147 is involved in cancer invasion and metastasis by inducing the expression of MMPs and affecting cell migration. In the present study, it was unexpected that the exogenous CD147-4 did not elevate the expression of MMPs and had no effects on cell migration (Fig. 5). We presumed that this was due to the structure of isoform- 4 with a distinct $\mathrm{N}$-terminus and lack of the first Ig domain; only high-glycosylated CD147 is responsible for activating the expression of MMPs $(30,31)$.

Taken together, as in other types of cancers, CD147 plays important roles in cervical cancers. In addition, CD147-4 may facilitate tumor progression through promoting cell proliferation. Nevertheless, further investigation is required to elucidate the molecular mechanisms of CD147-4 in cervical cancers.

\section{Acknowledgements}

We are grateful to Dr J.S. Tang for his helpful comments in preparing this manuscript. The project was supported by the National Natural Science Foundation of China (30571951/ C1702) and a grant of the Excellent Young Scientist (30725043/ C1702).

\section{References}

1. Miyauchi T, Kanekura T, Yamaoka A, Ozawa M, Miyazawa S and Muramatsu T: Basigin, a new, broadly distributed member of the immunoglobulin superfamily, has strong homology with both the immunoglobulin V domain and the beta-chain of major histocompatibility complex class II antigen. J Biochem 107: 316-323, 1990. 
2. Ellis SM, Nabeshima K and Biswas C: Monoclonal antibody preparation and purification of a tumor cell collagenasestimulatory factor. Cancer Res 49: 3385-3391, 1989.

3. Biswas C, Zhang Y, DeCastro R, et al: The human tumor cellderived collagenase stimulatory factor (renamed EMMPRIN) is a member of the immunoglobulin superfamily. Cancer Res 55: 434-439, 1995.

4. Xu J, Xu HY, Zhang Q, et al: HAb18G/CD147 functions in invasion and metastasis of hepatocellular carcinoma. Mol Cancer Res 5: 605-614, 2007.

5. Yan L, Zucker S and Toole BP: Roles of the multifunctional glycoprotein, emmprin (basigin; CD147), in tumour progression. Thromb Haemost 93: 199-204, 2005.

6. Tang Y, Nakada MT, Rafferty P, et al: Regulation of vascular endothelial growth factor expression by EMMPRIN via the PI3K-Akt signaling pathway. Mol Cancer Res 4: 371-377, 2006.

7. Sato T, Ota T, Watanabe M, Imada K, Nomizu M and Ito A: Identification of an active site of EMMPRIN for the augmentation of matrix metalloproteinase- 1 and -3 expression in a co-culture of human uterine cervical carcinoma cells and fibroblasts. Gynecol Oncol 114: 337-342, 2009.

8. Li M,Zhai Q, Bharadwaj U, et al: Cyclophilin A is overexpressed in human pancreatic cancer cells and stimulates cell proliferation through CD147. Cancer 106: 2284-2294, 2006.

9. Xu D and Hemler ME: Metabolic activation-related CD147CD98 complex. Mol Cell Proteomics 4: 1061-1071, 2005.

10. Fingar DC and Blenis J: Target of rapamycin (TOR): an integrator of nutrient and growth factor signals and coordinator of cell growth and cell cycle progression. Oncogene 23: 3151-3171, 2004.

11. Parkin DM, Bray F, Ferlay J and Pisani P: Global cancer statistics, 2002. CA Cancer J Clin 55: 74-108, 2005.

12. Baseman JG and Koutsky LA: The epidemiology of human papillomavirus infections. J Clin Virol 32 (Suppl 1): S16-S24, 2005.

13. Holmes RS, Hawes SE, Toure P, et al: HIV infection as a risk factor for cervical cancer and cervical intraepithelial neoplasia in Senegal. Cancer Epidemiol Biomarkers Prev 18: 2442-2446, 2009.

14. Jin JS, Hsieh DS, Lin YF, Wang JY, Sheu LF and Lee WH Increasing expression of extracellular matrix metalloprotease inducer in renal cell carcinoma: tissue microarray analysis of immunostaining score with clinicopathological parameters. Int J Urol 13: 573-580, 2006

15. Ji J and Zheng PS: Expression of Sox 2 in human cervical carcinogenesis. Hum Pathol 41: 1438-1447, 2010

16. Liu JC, Deng T, Lehal RS, Kim J and Zacksenhaus E: Identification of tumorsphere- and tumor-initiating cells in HER2/ Neu-induced mammary tumors. Cancer Res 67: 8671-8681, 2007.

17. Belton RJ Jr, Chen L, Mesquita FS and Nowak RA: Basigin-2 is a cell surface receptor for soluble basigin ligand. J Biol Chem 283: 17805-17814, 2008.
18. Reimers N, Zafrakas K, Assmann V, et al: Expression of extracellular matrix metalloproteases inducer on micrometastatic and primary mammary carcinoma cells. Clin Cancer Res 10: 3422-3428, 2004

19. Sameshima T, Nabeshima K, Toole BP, et al: Expression of emmprin (CD147), a cell surface inducer of matrix metalloproteinases, in normal human brain and gliomas. Int $\mathrm{J}$ Cancer 88: 21-27, 2000.

20. Kanekura T, Chen X and Kanzaki T: Basigin (CD147) is expressed on melanoma cells and induces tumor cell invasion by stimulating production of matrix metalloproteinases by fibroblasts. Int J Cancer 99: 520-528, 2002.

21. Ju XZ, Yang JM, Zhou XY, Li ZT and Wu XH: EMMPRIN expression as a prognostic factor in radiotherapy of cervical cancer. Clin Cancer Res 14: 494-501, 2008.

22. Peters WM: Nature of 'basal' and 'reserve' cells in oviductal and cervical epithelium in man. J Clin Pathol 39: 306-312, 1986.

23. Reya T, Morrison SJ, Clarke MF and Weissman IL: Stem cells, cancer, and cancer stem cells. Nature 414: 105-111, 2001.

24. Hanna SM, Kirk P, Holt OJ, Puklavec MJ, Brown MH and Barclay AN: A novel form of the membrane protein CD147 that contains an extra Ig-like domain and interacts homophilically. BMC Biochem 4: 17, 2003.

25. Sun J and Hemler ME: Regulation of MMP-1 and MMP-2 production through CD147/extracellular matrix metalloproteinase inducer interactions. Cancer Res 61: 2276-2281, 2001.

26. Ochrietor JD, Moroz TP, van Ekeris L, et al: Retina-specific expression of 5A11/Basigin-2, a member of the immunoglobulin gene superfamily. Invest Ophthalmol Vis Sci 44: 4086-4096, 2003.

27. Khanna A, Bockelman C, Hemmes A, et al: MYC-dependent regulation and prognostic role of CIP2A in gastric cancer. J Natl Cancer Inst 101: 793-805, 2009.

28. Junttila MR, Puustinen P, Niemela M, et al: CIP2A inhibits PP2A in human malignancies. Cell 130: 51-62, 2007.

29. Eckerdt F, Yuan J and Strebhardt K: Polo-like kinases and oncogenesis. Oncogene 24: 267-276, 2005.

30. Tang W, Chang SB and Hemler ME: Links between CD147 function, glycosylation, and caveolin-1. Mol Biol Cell 15: 4043-4050, 2004.

31. Tang W and Hemler ME: Caveolin-1 regulates matrix metalloproteinases-1 induction and CD147/EMMPRIN cell surface clustering. J Biol Chem 279: 11112-11118, 2004.

32. Prince ME, Sivanandan R, Kaczorowski A, et al: Identification of a subpopulation of cells with cancer stem cell properties in head and neck squamous cell carcinoma. Proc Natl Acad Sci USA 104: 973-978, 2007. 